\title{
Caracterização do progresso da queima das folhas em diferentes genótipos de cenoura
}

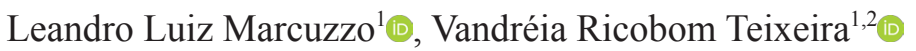

${ }^{1}$ Instituto Federal Catarinense - IFC/Campus Rio do Sul, CP 441, CEP 89.163-356, Rio do Sul, SC, Brasil. ${ }^{2}$ Aluna do curso de especialização em sistemas produtivos regionais do IFC/Campus Rio do Sul, CP 441, CEP 89.163-356, Rio do Sul, SC, Brasil. e-mail: ricobom.13@hotmail.com Autor para correspondência: Leandro Luiz Marcuzzo (leandro.marcuzzo@ifc.edu.br)

Data de chegada: 08/11/2017. Aceito para publicação em: 05/02/2019.

\section{RESUMO}

$10.1590 / 0100-5405 / 189666$

Marcuzzo, L.L.; Teixeira, V.R. Caracterização do progresso da queima das folhas em diferentes genótipos de cenoura. Summa Phytopathologica, v.45, n.2, p.219-222, 2019.

A cultura da cenoura tem se intensificado devido ao consumo in natura e também pelo processamento industrial de conservas. Entretanto, a queima das folhas (Alternaria dauci) tem comprometido a produtividade. Como não se conhece o comportamento epidemiológico da doença nas condições de sul de Brasil, esse trabalho teve como objetivo analisar a epidemiologia temporal da doença em diferentes genótipos de cenoura. Em delineamento experimental de blocos casualizados com quatro repetições foram avaliados os genótipos Alvorada calibrada média; Brasília; Brazlândia, Ferracini, Flakee; Nantes; Nantes Express e Suprema calibrada média. Para avaliação da severidade da doença foi utilizada uma escala descritiva em dez plantas ao acaso previamente demarcadas. A curva de progresso da doença para cada um dos genótipos foi ajustada ao modelo de Gompertz, já que a severidade observada correspondeu ao modelo e foi confirmada pela coerência entre os pontos estimados e pelo resíduo. Foi verificado que o genótipo Suprema apresentou a maior taxa $(0,533)$ de progresso, enquanto que Brazlândia a menor $(0,368)$. Verificou-se que o genótipo Nantes apresentou o pico máximo da severidade com $66,9 \%$ e que Suprema teve o menor acúmulo da doença com $48,2 \%$. Os genótipos não apresentaram diferenças no comportamento do progresso à queima das folhas da cenoura.

Palavras-chave: Daucus carota, genótipos, epidemiologia, Alternaria dauci.

\section{ABSTRACT}

Marcuzzo, L.L.; Teixeira, V.R. Characterization of the progress of carrot leaf spot in different carrot genotypes. Summa Phytopathologica, v.45, n.2, p.219-222, 2019.

Carrot cultivation has intensified due to in natura consumption and industrial canning processing. However, carrot leaf spot (Alternaria dauci) has compromised the productivity. As the epidemiological behavior of this disease in southern Brazil is not known, the present study aimed to analyze its temporal epidemiology in different carrot genotypes. In an experimental design of randomized blocks and four replicates, the following genotypes were evaluated: "Alvorada calibrada média"; "Brasília"; "Brazlândia"; "Ferracini"; "Flakee"; "Nantes"; "Nantes Express" and "Suprema calibrada média". To evaluate the disease severity, a diagrammatic scale was used for ten plants previously demarcated at random. The disease progress curve for each genotype was adjusted to Gompertz model, since the observed severity corresponded to the model and was confirmed by the consistency between estimated points and the residue. The genotype "Suprema" showed the highest progress rate (0.533), while "Brazlândia" $(0.368)$ showed the lowest rate. "Nantes" had the highest severity peak, $66.9 \%$, and "Suprema" had the lowest disease accumulation, $48.2 \%$. The genotypes had no differences in their behaviors for temporal progress of carrot leaf spot.

Keywords: Daucus carota, genotypes, epidemiology, Alternaria dauci.

A cenoura (Daucus carota L.) é amplamente cultivada no Brasil, porém a ocorrência de doenças é um fator limitante para a produção da cultura. Entre as doenças, a queima das folhas causada por Alternaria dauci (Kühn) Groves \& Skolko, tem sido considerada a mais destrutiva nas épocas mais quentes com temperatura entre 16 a $25^{\circ} \mathrm{C}$ e úmidas do ano $(4,7)$. A doença inicia-se nas folhas mais velhas e baixeiras, apresentando lesões marrom-esverdeadas, de aspecto encharcado e com a evolução dos sintomas, os tecidos atacados tornam-se marrom-escuros a pretos, podendo ser circundados por halos amarelos (10).

O uso de genótipos resistentes é uma das formas de manejo dessas doenças, porém é necessária a avaliação do comportamento temporal da doença entre genótipos. Entre as formas de caracterizar o desenvolvimento de fitopatógenos, a curva de progresso temporal é a melhor representação de uma epidemia. A interpretação do formato dessas curvas e seus componentes, como a taxa e a severidade final podem ser usadas para determinar o progresso da doença de genótipos $(1,2)$.
Como não se dispõe de informação sobre o assunto, o objetivo deste trabalho foi caracterizar o progresso temporal da queima das folhas em diferentes genótipos de cenoura.

O experimento foi implantado em 15 de setembro a 26 de dezembro de 2017 no Instituto Federal Catarinense, Campus de Rio do Sul, município de Rio do Sul/SC com latitude Sul de $27^{\circ} 11^{\prime} 07^{\prime}$ ', longitude Oeste de 49³9’39” e altitude de 687 metros do nível do mar.

Os dados meteorológicos foram obtidos de uma estação Davis ${ }^{\mathbb{R}}$ Vantage Vue $300 \mathrm{~m}$ localizado ao lado do experimento e os dados médios durante a condução do experimento foram de $19,7^{\circ} \mathrm{C}$ para temperatura do ar, de 13,1 horas de umidade relativa do ar $\geq 90 \%$ e a precipitação pluvial acumulada foi de $579,8 \mathrm{~mm}$.

Sementes de cenoura dos genótipos: Alvorada calibrada média, Brasília, Brazlândia, Ferracini, Flakee, Nantes, Nantes Express e Suprema calibrada média foram semeadas a campo em quatro repetições constituídas de uma área de 1,5 X 1,0 m utilizando quatro linhas com 
Tabela 1. Parâmetros estimados pelo modelo de Gompertz e ajustado aos dados da queima das folhas em diferentes genótipos de cenoura. IFC/ Campus Rio do Sul, 2017

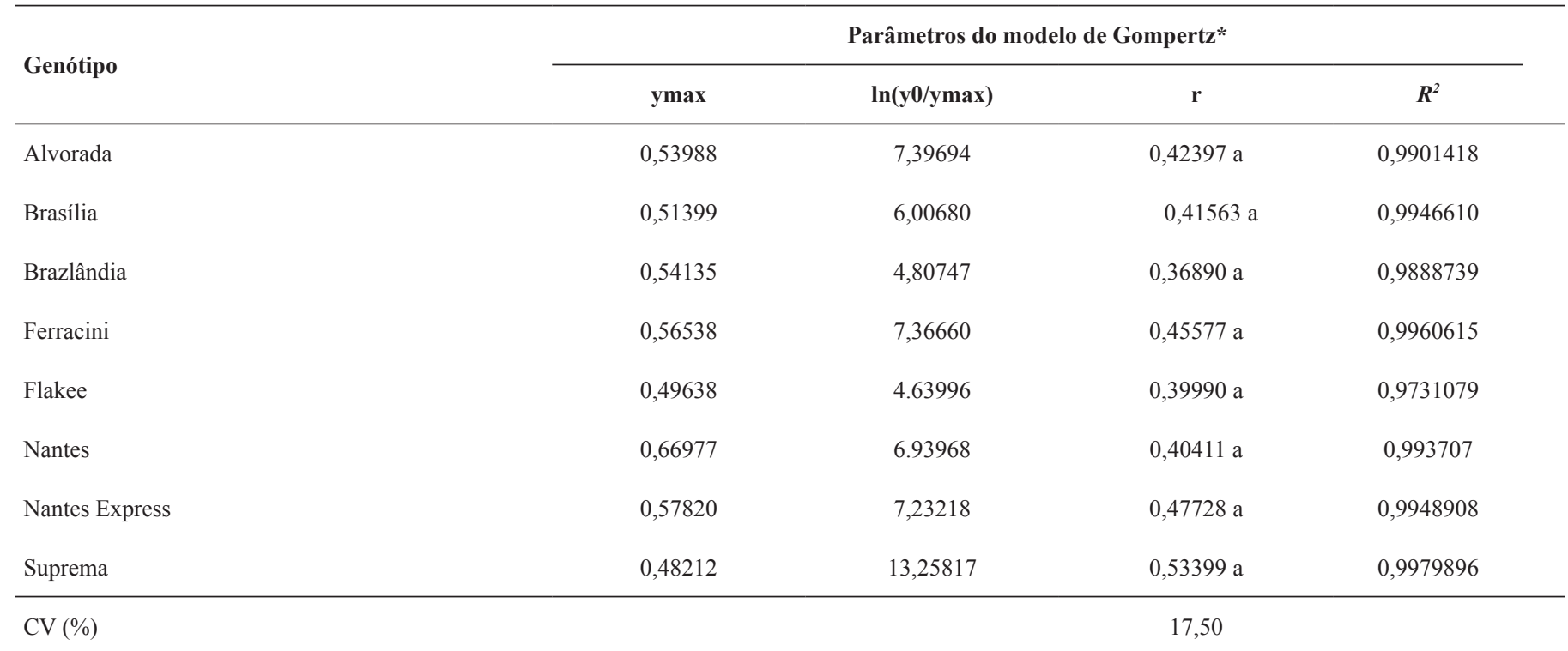

*y $=$ ymax * $(\exp (-\ln (\mathrm{y} 0 / \mathrm{ymax}) * \exp (-\mathrm{r} * \mathrm{x})))$, onde $\mathrm{y}$ : severidade estimada final $(\%$ de severidade/100); ymax: severidade máxima de doença/100; ln(y0/ymax $)$ refere-se a função de proporção da doença na primeira observação; $r$ : taxa, x o tempo em semanas e $R^{2}$ : coeficiente de determinação. Médias seguidas de mesma letra não diferem estatisticamente pelo teste de agrupamento de Scott-Knott ao nível de 5\%. CV- coeficiente de variação.

espaçamento de $0,25 \mathrm{~m}$ entre linhas e após o raleio (25 dias após a semeadura), deixando-se uma planta a cada $4 \mathrm{~cm}$ (equivalente a 1.000.000 plantas.ha ${ }^{-1}$ ), ficando com um estande final de 150 plantas em cada repetição.

Para que houvesse inóculo do patógeno na área, mudas de cenoura Nantes (considerada suscetível a doença) com 30 dias de idade foram inoculadas com auxílio de um atomizador portátil uma suspensão $\left(10^{4}\right)$ de conídios de $A$. dauci e após 24 horas de câmara úmida foram transplantadas a cada um metro linear ao redor do experimento no dia da semeadura.

Para confirmação do agente etiológico da doença no momento da avaliação, já que é um complexo de patógenos que causa a queima das folhas (7), algumas folhas foram acondicionadas em câmara úmida por 48 horas e após isso foi visualizado apenas conídios de Alternaria dauci em microscópio ótico.

Semanalmente, após a semeadura, foi avaliado a severidade da queima das folhas em 10 plantas demarcadas aleatoriamente em cada repetição, com auxílio de escala descritiva proposta por Souza et al. (9).

Modelos não lineares, comumente usados para representar crescimento de epidemias como o Logístico e o de Gompertz foram usados para ajuste com os dados observados utilizando o software $\mathrm{R}$ versão 2.15.1(6). Os critérios estabelecidos para comparação dos modelos, em função da qualidade do ajustamento dos dados foram: a) erro padrão da estimativa; b) estabilidade dos parâmetros; c) erro padrão dos resíduos; d) visualização da distribuição dos resíduos ao longo do tempo e e) coeficiente de determinação $\left(\mathrm{R}^{2}\right)$.

Os dados da taxa foram submetidos à análise de variância pelo teste de $\mathrm{F}$ a $5 \%$, e se significativos, as médias foram comparadas estatisticamente pelo teste de agrupamento de Scott-Knott ao nível de 5\% pelo software estatístico SASM-Agri (3) para avaliar se há diferença(s) entre o genótipo quanto a taxa de progresso da doença.

O modelo de Gompertz expresso por $\mathrm{y}=\mathrm{ymax} *(\exp (-\ln (\mathrm{y} 0 / \mathrm{ymax})$ $* \exp (-r * x)))$, onde y: severidade estimada final $(\%$ de severidade $/ 100)$; ymax: severidade máxima de doença/100; $\ln (\mathrm{y} 0 /$ ymax $)$ refere-se a função de proporção da doença na primeira observação; r: taxa,x o tempo em semanas após o inicio da doença foi escolhido para representar o progresso da queima das folhas na avaliação dos genótipos de cenoura (Tabela 1), devido a queima das folhas ser considerada uma doença explosiva após o inicio dos primeiros sintomas. Possivelmente todos os dados se ajustaram em decorrência de ser a época de cultivo da cultura e a presença da doença no local.

A análise dos dados e as equações originadas pelo modelo de Gompertz (Tabela 1) resultaram em um coeficiente de determinação significativo, e a severidade observada correspondeu ao modelo, confirmada pela coerência entre os pontos estimados e do resíduo (erro) nas oito semanas de avaliação (Figura 1).

A taxa de progresso da doença não apresentou diferenças estatísticas entre os genótipos (Tabela 1). Foi verificado que o genótipo Suprema apresentou a maior taxa $(0,533)$ de progresso, enquanto que Brazlândia a menor $(0,368)$. Constatou-se que o genótipo Nantes apresentou o pico máximo da severidade com $66,9 \%$ e que Suprema teve o menor acúmulo da doença com 48,2\% com uma diferença de $27,95 \%$ na severidade máxima entre esses dois genótipos (Tabela 1).

Souza et al. (9) também encontrou valores de intensidade próximo dos $60 \%$ em Nantes como verificado nesse trabalho (Figura 1). Pereira et al. (5) constataram que o genótipo Brasília apresentou superioridade no acumulado da doença quando comparado a outros 27 genótipos oriundos de cruzamentos. Fato esse foi confirmado por Shibata (8) quando avaliou a reação de 13 genótipos de cenoura, constatou superioridade na doença no genótipo Brasília em relação à Alvorada e Brazlândia. No entanto, esse comportamento para Brasília não foi verificado para as condições de cultivo nessa época na região do alto vale do Itajaí.

Conclui-se que os genótipos avaliados não apresentaram diferença no comportamento de progresso temporal da queima das folhas causado por Alternaria dauci. 


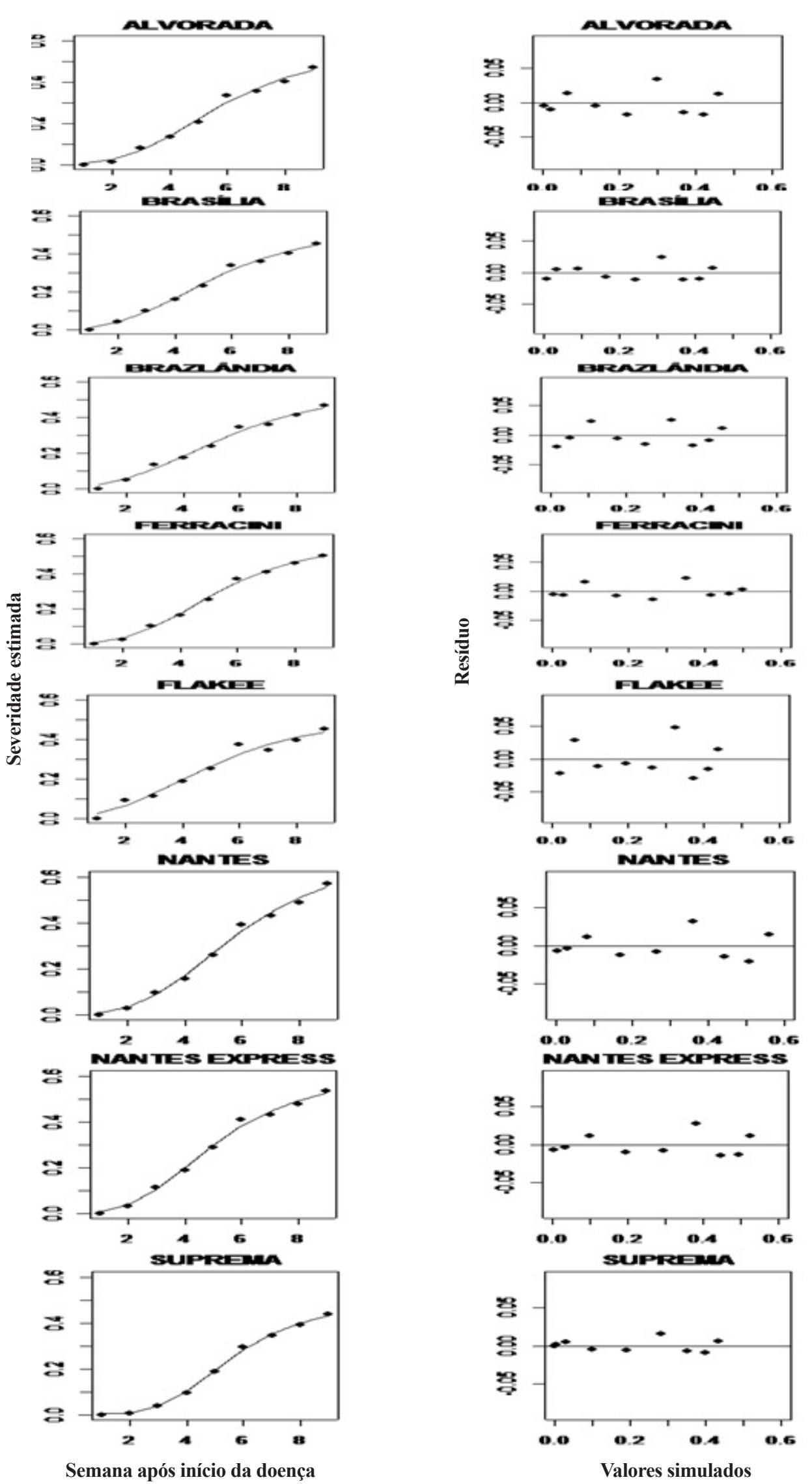

Figura 1. Curva da severidade estimada do progresso da queima das folhas em diferentes genótipos de cenoura e seus respectivos resíduos ajustados pelo modelo de Gompertz. IFC/Campus Rio do Sul, 2017. 


\section{REFERÊNCIAS}

1. Bergamim Filho, A.; Amorim, L. Doenças de plantas tropicais: epidemiologia e controle econômico. São Paulo: Ceres, 1996, 289p.

2. Campbell, C.L.; Madden, L.V. Introduction to plant disease epidemiology. New York : Wiley Interscience, 1990. 532p.

3. Canteri, M.G.; Althaus, R.A.; Virgens Filho, J.S.; Giglioti, E.A.; Godoy, C.V. SASM - Agri: Sistema para análise e separação de médias em experimentos agrícolas pelos métodos Scoft - Knott, Tukey e Duncan. Revista Brasileira de Agrocomputação, Ponta Grossa, v.1, p.18-24, 2001.

4. Henz, G.P.; Lopes, C.A.; Reis, A. Manejo de Doenças. In: Nick, C.; Borém, A. Cenoura: do plantio a colheita. UFV: Viçosa, p.98-123, 2016.

5. Pereira, R.B; Carvalho, A.D.F; Pinheiro, J.B; Silva, G.O; Vieira, J.V. Resistencia de populações de cenoura à queima-das-folhas com diferentes níveis de germoplasma tropical. Horticultura brasileira, Brasília, DF, v.30, n.3, p489-493, 2012
6. R Development Core Team (2012). R: A language and environment for statistical computing. R Foundation for Statistical Computing, Vienna, Austria. Disponível em: <http://www.R-project.org>. Acesso em: $28 \mathrm{dez}$. 2017.

7. Reis, A. Queima das folhas: uma doença complexa. Brasília: $\mathrm{CNPH}$, 2010, 8p. Circular técnica 91

8. Shibata, E. T. Reação de cultivares de cenoura à queima das folhas, prevalência de patógenos, diversidade genética e sensibilidade de Alternaria dauci a fungicidas. 2008.98 f. Dissertação (Mestrado Fitopatologia). Universidade de Brasília, Brasília.

9. Souza, R.T.; Forcelini, C.A.; Reis, E.M.; Calvete, E.O. Validação de dois sistemas de previsão para a queima das folhas da cenoura. Fitopatologia Brasileira, Brasília, DF, v.27, n.2, p.87-90, 2002.

10. Töfoli, J.G.; Domingues, R.J. Sintoma, etiologia e manejo da queima das folhas (Alternaria dauci; Cercospora carotae) na cultura da cenoura. Biológico, São Paulo, v.72, n.1, p.47-50, 2010. 